\title{
Theoretical Analysis of Power Saving in Cognitive Radio with Arbitrary Inputs
}

\author{
Ahmed Sohail, Mohammed Al-Imari, Pei Xiao, and Barry G. Evans
}

\begin{abstract}
In Orthogonal Frequency Division Multiplexing (OFDM) based cognitive radio systems, power optimization algorithms have been evaluated to maximize the achievable data rates of the Secondary User (SU). However, unrealistic assumptions are made in the existing work, i.e. a Gaussian input distribution and traditional interference model that assumes frequency division multiplexing modulated Primary User (PU) with perfect synchronization between the PU and the SU. In this paper, we first derive a practical interference model by assuming OFDM modulated PU with imperfect synchronization. Based on the new interference model, the power optimization problem is proposed for the Finite Symbol Alphabet (FSA) input distribution (i.e., M-QAM), as used in practical systems. The proposed scheme is shown to save transmit power and to achieve higher data rates compared to the Gaussian optimized power allocation and the uniform power loading schemes. Furthermore, a theoretical framework is established in this paper to estimate the power saving by evaluating optimal power allocation for the Gaussian and the FSA input. Our theoretical analysis is verified by simulations and proved to be accurate. It provides guidance for the system design and gives deeper insights into the choice of parameters affecting power saving and rate improvement.
\end{abstract}

Index Terms-Cognitive Radio, OFDM, Finite Symbol Alphabet, MMSE, Mutual Information.

\section{INTRODUCTION}

Cognitive Radio (CR) technology [1] plays a significant role in making the best use of scarce spectrum to meet the increasing demand for emerging wireless applications, e.g., public safety, broadband cellular, and the medical body area networks for medical applications [2]. CR technology also plays a potential role in vehicular communications in terms of safety applications and fulfilling the growing demand and usage of in-car entertainment [3]. In the interweave spectrum sharing scheme of the CR system, where the Primary User (PU) and the Secondary User (SU) co-exist in adjacent frequency bands, mutual interference is a limiting factor on the performance of both the PU and the SU. This can be dealt by dynamic power allocation schemes in Orthogonal Frequency Division Multiplexing (OFDM) based CR systems by adjusting the transmit power on each subcarrier of the SU. Different power allocation schemes have been presented in the literature to maximize the SU data rate under the interference constraint, assuming the Gaussian input distribution [4][6]. The Gaussian input is theoretically optimal for Mutual Information (MI) maximization, however, it is not a valid assumption for practical systems and the power optimized for

The authors are with the Centre for Communication Systems Research, University of Surrey, Guildford, Surrey, GU2 7XH, UK (Email:\{a.sohail, m.alimari, p.xiao,b.evans\}@ surrey.ac.uk). the Gaussian input is suboptimal when it is used for Finite Symbol Alphabet (FSA) transmission. On the other hand, the FSA input distribution is a more realistic assumption for practical systems. Based on the fact that the MI attained by the FSA input is always lower than the capacity attained by the Gaussian input, the difference in achievable rate can be approximated by using a SNR gap model as proposed in [7]. However, the approximation is not valid at high SNRs due to the large gap.

One possible solution to address this limitation is to derive the optimal power with the FSA input, as given in [8], where authors only considered a non-cognitive scenario. To the best of our knowledge, no work has been done to derive an optimal power with FSA inputs in CR systems. Therefore, in [9], we derived the optimal power allocation for the FSA input in OFDM based CR systems. Through Monte Carlo simulations, we showed that there is a wastage of transmit power if the Gaussian optimized power is used for the FSA transmission. Whereas, the optimal power allocation derived by the proposed scheme leads to a significant power saving, i.e., $12-90 \%$, depending on the modulation scheme (i.e., BPSK, QPSK and 16-QAM) used. In the literature and in [9], interference from the secondary system to the primary system is calculated based on the assumption that (i) the SU and the PU are OFDM and FDM modulated, respectively, and (ii) both the PU and the SU are perfectly synchronized. In practical systems, these assumptions are unrealistic, since current wireless communication systems are OFDM modulated. Also it is difficult to maintain perfect synchronization. This paper is an extension of our previous work in [9] and the novel contributions of this paper are summarized below

- In this paper, Sec. II presents a novel practical interference model that assumes OFDM modulation scheme for both the PU and the SU with imperfect synchronization. This has not been reported in the literature. The previous interference model is only applicable to FDM modulation scheme. Based on the proposed interference model, the optimal power is derived for the FSA input distribution by capitalizing on the relationship between MI and MMSE [10] in Sec. III.

- Motivated by the results obtained in Sec. IV, we evaluate theoretically the average optimal power for the Gaussian and the FSA input, and accordingly calculate the power saving, which again has not been reported in the existing literature. Given channel statistics, the theoretical analysis can be used to estimate the power saving without running time consuming Monte Carlo simulations. In addition, it 
provides us a deeper insight into the parameters affecting power saving (i.e., the optimal power for the FSA input distribution is inversely proportional to $d^{2}$, where $d$ is the minimum distance for unit variance constellations.)

- Our theoretical analysis is validated by simulations in Sec. V and proves to be accurate. Furthermore, we compare achievable data rate for the FSA transmission under the optimal power allocation with FSA inputs and uniform power loading scheme [4]. We found that our proposed power allocation scheme outperforms the uniform power loading scheme.

The remainder of the paper is organized as follows. Secs. II, III and IV present the interference model, the optimal power allocation policy and theoretical analysis of power saving for OFDM based CR systems, respectively. We present the simulation and theoretical results of the proposed scheme in Sec. V. Finally, conclusions are drawn in Sec. VI.

\section{INTERFERENCE MODEL}

The system model can be found in [9]. We assume OFDM modulation scheme for both the SU and the PU with imperfect synchronization as shown in Fig. 1. Side-lobes are omitted in the figure for simplicity. Consider a frequency offset $\delta f$ such that $\epsilon=\frac{\delta f}{\Delta f}$, where $\epsilon$ is the normalized frequency offset and $\Delta f$ is subcarrier spacing. The SU samples with $\epsilon$ after Inverse Fast Fourier Transform (IFFT) are

$$
X_{k}^{s}=\frac{1}{N} \sum_{n_{s}=1}^{N} x_{n_{s}}^{s} e^{\frac{j 2 \pi\left(n_{s}+\epsilon\right) k}{N}}, \quad k=\{1, \ldots . N\} .
$$

The PU samples after IFFT are

$$
X_{k}^{p}=\frac{1}{N} \sum_{n_{p}=N+1}^{2 N} x_{n_{p}}^{p} e^{\frac{j 2 \pi n_{p} k}{N}}, \quad k=\{1, \ldots . N\},
$$

where $2 N$ is total number of subcarriers, subscripts $s, p$ represent SU and PU, respectively, and $k$ is number of time samples. Given $\epsilon$ and omitting the channel effect and noise, the input of the Fast Fourier Transform (FFT) for the PU and the $\mathrm{SU}$ is given by

$$
y_{k}=\frac{1}{N} \sum_{n_{s}=1}^{N} x_{n_{s}}^{s} e^{\frac{j 2 \pi\left(n_{s}+\epsilon\right) k}{N}}+\frac{1}{N} \sum_{n_{p}=N+1}^{2 N} x_{n_{p}}^{p} e^{\frac{j 2 \pi n_{p} k}{N}} .
$$

Consider the $\ell$ th output of the PU FFT, $\left(Y_{\ell}^{p}\right)$ which corresponds to the symbol received on the $\ell$ th subcarrier. This is given as

$$
Y_{\ell}^{p}=\sum_{k=1}^{N} y_{k} e^{\frac{-j 2 \pi k \ell}{N}}, \quad \ell=\{N+1, \ldots .2 N\} .
$$

By substituting Eq. (3) into Eq. (4), and after mathematical manipulations, we obtain

$$
\begin{aligned}
Y_{\ell}^{p}= & \frac{1}{N} \sum_{k=1}^{N} \sum_{n_{s}=1}^{N} x_{n_{s}}^{s} e^{\frac{j 2 \pi\left(n_{s}-\ell+\epsilon\right) k}{N}}+X_{\ell}^{p} \\
& +\frac{1}{N} \sum_{k=1}^{N} \sum_{n_{p}=N+1 ; n_{p} \neq \ell}^{2 N} x_{n_{p}}^{p} e^{\frac{j 2 \pi\left(n_{p}-\ell\right) k}{N}} .
\end{aligned}
$$

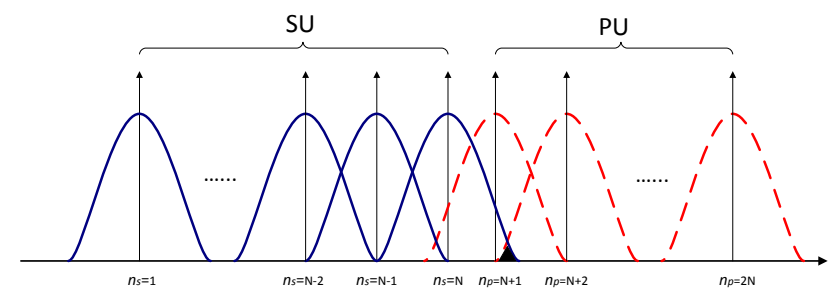

Fig. 1. Graphical representation of OFDM modulated PU and SU with imperfect synchronization

Based on Eq. (5), it can be easily shown that

$$
Y_{\ell}^{p}=X_{\ell}^{p}+\frac{1}{N} \sum_{k=1}^{N} \sum_{n_{s}=1}^{N} x_{n_{s}}^{s} e^{\frac{j 2 \pi\left(n_{s}-\ell+\epsilon\right) k}{N}} .
$$

In Eq. (6), the second term is the net interference to the $\ell$ th subcarrier of the PU from all the SU subcarriers and is denoted by $\psi_{\ell}$

$$
\psi_{\ell}=\frac{1}{N} \sum_{k=1}^{N} \sum_{n_{s}=1}^{N} x_{n_{s}}^{s} e^{\frac{j 2 \pi\left(n_{s}-\ell+\epsilon\right) k}{N}} .
$$

Define $\psi_{\ell, n_{s}}$ as the interference from the $n_{s}$ th SU subcarrier to the $\ell$ th PU subcarrier, i.e.,

$$
\psi_{\ell, n_{s}}=\frac{1}{N} x_{n_{s}}^{s} \sum_{k=1}^{N} e^{\frac{j 2 \pi\left(n_{s}-\ell+\epsilon\right) k}{N}} .
$$

Note that the signal spectra of each subcarrier is a Sinc function, therefore the interference calculation expressed in Eq. (8) has taken the side-lobes of the Sinc function into account. The average $\bar{\psi}_{\ell, n_{s}}$ can thus be calculated as

$$
\bar{\psi}_{\ell, n_{s}}=\int_{0}^{\epsilon_{\max }} \frac{1}{N} x_{n_{s}}^{s} \sum_{k=1}^{N} e^{\frac{j 2 \pi\left(n_{s}-\ell+\epsilon\right) k}{N}} d \epsilon .
$$

After mathematical manipulations, we obtain

$$
\bar{\psi}_{\ell, n_{s}}=\frac{j x_{n_{s}}^{s}}{2 \pi} \sum_{k=1}^{N} \frac{1}{k}\left[e^{\frac{j 2 \pi\left(n_{s}-\ell\right) k}{N}}-e^{\frac{j 2 \pi\left(n_{s}-\ell+\epsilon\right) k}{N}}\right] .
$$

Interference power can be calculated as $J_{\ell, n_{s}}=$ $E_{x_{n_{s}}^{s}}\left|\bar{\psi}_{\ell, n_{s}}\right|^{2}=p_{n_{s}} \Phi_{\ell, n_{s}}$, where $p_{n_{s}}$ is the transmit power of the $n_{s}$ th SU subcarrier and

$$
\Phi_{\ell, n_{s}}=\left|\left[\frac{j}{2 \pi} \sum_{k=1}^{N} \frac{1}{k}\left[e^{\frac{j 2 \pi\left(n_{s}-\ell\right) k}{N}}-e^{\frac{j 2 \pi\left(n_{s}-\ell+\epsilon\right) k}{N}}\right]\right]\right|^{2} .
$$

\section{Optimal Power Allocation Policy}

The objective of the power optimization is to calculate an optimal power with FSA input that maximizes the MI of the SU under given constraints, which formulated as follows

$$
\max _{p_{n_{s}}} \sum_{n_{s}=1}^{N} I\left(p_{n_{s}} g_{n_{s}}\right)
$$


subject to

$$
\sum_{n_{s}=1}^{N} p_{n_{s}} \Phi_{\ell, n_{s}}=\frac{\tau_{t h}}{\Omega}, \quad \text { and } p_{n_{s}} \geq 0, \quad n_{s}=1, \cdots, N
$$

where $\tau_{t h}$ is the interference threshold prescribed by the PU, $g_{n_{s}}$ is the channel gain between the SU transmitter and receiver of the $n_{s}$ th subcarrier and $\Omega$ is the path loss between the SU transmitter and the PU receiver. In the rest of the paper, $n_{s}, p_{n_{s}}, g_{n_{s}}$ and $\Phi_{\ell, n_{s}}$ is represented as $n, p_{n}, g_{n}$ and $\Phi_{n}$, respectively, whenever no ambiguity arises. In [9], the optimal power is derived and is given as

$$
p_{n}^{\star}= \begin{cases}\frac{1}{g_{n}} \mathrm{mmse}^{-1}\left(\frac{\lambda \Phi_{n}}{g_{n}}\right) & \text { if } \frac{g_{n}}{\Phi_{n}}>\lambda \\ 0 & \text { if } \frac{g_{n}}{\Phi_{n}} \leq \lambda\end{cases}
$$

where $\lambda$ is the Lagrange multiplier for the interference constraint and can be solved using numerical methods, (such as bisection, secant, or Newton) by solving the following equation

$$
\sum_{n=1}^{\left(N, \frac{g_{n}}{\Phi_{n}}>\lambda\right)} \frac{1}{g_{n}} \mathrm{mmse}^{-1}\left(\frac{\lambda \Phi_{n}}{g_{n}}\right) \Phi_{n}-\frac{\tau_{t h}}{\Omega}=0 .
$$

Simulation results are presented in Sec. V. We denote the total transmit optimal power $\left(P^{\star}=\sum_{n=1}^{N} p_{n}^{\star}\right)$ with Gaussian inputs as $P_{G}^{\star}$ and with FSA inputs as $P_{\mathcal{F}}^{\star}$. In Fig. 2 and Fig. 3, we plot optimal power allocation and percentage of power saving, [i.e., $\left(\left(P_{G}^{\star}-P_{\mathcal{F}}^{\star}\right) / P_{G}^{\star}\right) \times 100$ ] in CR systems using Monte Carlo simulations. In our simulations, we have adopted LTE parameters for the SU transmission and assume that a total of $10 \mathrm{MHz}$ bandwidth is divided into 50 Resource Blocks (RBs) [11]. We consider a simplified path loss model, i.e., $Q\left(r_{0} / r\right)^{\gamma}$ [12] for the simulations, where $Q$ is constant, $\gamma$ is path loss exponent, $r_{0}$ (reference distance) and $r$ (distance between the SU transmitter and the PU receiver) are defined in meters. The values of $\epsilon, \gamma$ and $r_{0}$ are $0.04,2.7$ (for urban microcells) and $50 \mathrm{~m}$, and $\tau_{t h}$ is assumed to be equivalent to thermal noise per RB, respectively. The interference introduced to the PU changes according to $r$ which is assumed to vary from $50-85 \mathrm{~m}$. We adopt the IEEE 802.11 multipath channel model with root mean square delay spread of $50 \mathrm{~ns}$. The results are averaged over 2000 snapshots. It can be clearly seen from Fig. 3 that a significant power saving has been achieved by the proposed optimal power $P_{\mathcal{F}}^{\star}$ in comparison to $P_{G}^{\star}$. The transmit power saving for distances ranging from $50-85 \mathrm{~m}$ has found to be $65-90 \%, 49.5-83 \%$ and $12-60 \%$ for BPSK, QPSK and 16-QAM inputs, respectively.

\section{Theoretical Analysis of Power SaVing}

Motivated by promising power saving results, in this section we theoretically analyse the power saving. The advantage is that, for given channel statistics, the theoretical analysis can be used to estimate the power saving without running time consuming Monte Carlo simulations.

Theorem 1: The power saving for a Rayleigh channel distribution by using the proposed optimal power $\left(P_{\mathcal{F}}^{*}\right)$ compared to conventional power allocation scheme $\left(P_{G}^{*}\right)$ is given by

$$
\bar{P}_{\text {saving }}^{*}=\bar{P}^{*}(G)-\bar{P}^{*}(\mathcal{F}),
$$

where

$$
\bar{P}^{*}(G)=\sum_{n=1}^{N}\left[\frac{\Gamma\left(1, \frac{\Phi_{n}^{2} \lambda^{2}}{2 \sigma^{2}}\right)}{\lambda \Phi_{n}}-\frac{\Gamma\left(\frac{1}{2}, \frac{\Phi_{n}^{2} \lambda^{2}}{2 \sigma^{2}}\right)}{\sqrt{2} \sigma}\right]
$$

and

$$
\begin{aligned}
\bar{P}^{*}(\mathcal{F}) \approx & \frac{\sqrt{2}}{d^{2} \sigma} \sum_{n=1}^{N}\left[A \Gamma\left(\frac{1}{2}, \frac{\Phi_{n}^{2} \lambda^{2}}{2 \sigma^{2}}\right)+\sqrt{2} B \sigma \Gamma\left(1, \frac{\Phi_{n}^{2} \lambda^{2}}{2 \sigma^{2}}\right)\right. \\
& \left.+2 C \sigma^{2} \Gamma\left(\frac{3}{2}, \frac{\Phi_{n}^{2} \lambda^{2}}{2 \sigma^{2}}\right)+2 \sqrt{2} D \sigma^{3} \Gamma\left(2, \frac{\Phi_{n}^{2} \lambda^{2}}{2 \sigma^{2}}\right)\right]
\end{aligned}
$$

where

$$
\begin{aligned}
& A=\left(f(a)-a f^{\prime}(a)+\frac{a^{2} f^{\prime \prime}(a)}{2}-\frac{a^{3} f^{\prime \prime \prime}(a)}{6}\right) ; \\
& B=\left(f^{\prime}(a)-a f^{\prime \prime}(a)+\frac{a^{2} f^{\prime \prime \prime}(a)}{2}\right) ; \\
& C=\left(\frac{f^{\prime \prime}(a)}{2}-\frac{a f^{\prime \prime \prime}(a)}{2}\right) ; \\
& D=\frac{f^{\prime \prime \prime}(a)}{6}, \quad f(a)=W\left(\alpha_{n} a^{2}\right),
\end{aligned}
$$

and $f^{\prime}(a)$ denotes the derivative of $f$ evaluated at point $a, \sigma$ is the channel statistic parameter for Rayleigh distribution, $\Gamma($. is the incomplete gamma function [13] and $d$ is the minimum distance for unit variance constellations, i.e., $d=2, \sqrt{2}$ and $\sqrt{2 / 5}$ for BPSK, QPSK and 16-QAM, respectively.

Proof: The average optimal power for a given $\lambda$ with arbitrary input distributions can be obtained as

$$
\bar{P}^{\star}(S)=\sum_{n=1}^{N} \int_{\Phi_{n} \lambda}^{\infty} p^{\star}\left(g_{n}, S\right) h\left(g_{n}\right) d g_{n},
$$

where $h\left(g_{n}\right)$ is a pdf of the channel, and for a Rayleigh fading channel $h\left(g_{n}\right)=\left(g_{n} / \sigma^{2}\right) e^{\left(-g_{n}^{2} / 2 \sigma^{2}\right)}$. The MMSE relationships for FSA and Gaussian input distributions are given by [8]

$$
\begin{gathered}
\operatorname{mmse}_{(\mathcal{F})}\left(p_{n}^{\star} g_{n}\right) \approx U \frac{e^{\frac{-d^{2}}{4}\left(p_{n}^{\star} g_{n}\right)}}{\sqrt{p_{n}^{\star} g_{n}}}, \\
\operatorname{mmse}_{(G)}\left(p_{n}^{\star} g_{n}\right)=\frac{1}{1+p_{n}^{\star} g_{n}},
\end{gathered}
$$

where $U=\frac{\sqrt{\pi}}{d}$ and 1 for M-PSK and M-QAM, respectively. To calculate $p_{n}^{\star}\left(g_{n}, \mathcal{F}\right)$ and $p_{n}^{\star}\left(g_{n}, G\right)$, we substitute Eqs. (21) and (22) into Eq. (14). After some mathematical manipulations, we obtain

$$
\begin{gathered}
e^{\frac{d^{2}}{4}\left(p_{n}^{\star} g_{n}\right)} \sqrt{p_{n}^{\star}}=\frac{U \sqrt{g_{n}}}{\lambda \Phi_{n}}, \\
p_{n}^{\star}\left(g_{n}, \mathcal{F}\right)=\frac{2}{d^{2} g_{n}} W\left(\frac{U^{2} d^{2} g_{n}^{2}}{2 \lambda^{2} \Phi_{n}^{2}}\right), \\
p_{n}^{\star}\left(g_{n}, G\right)=\frac{1}{\lambda \Phi_{n}}-\frac{1}{g_{n}},
\end{gathered}
$$


where $W($.$) is the Lambert \mathrm{W}$ function [14]. From Eq. (20), the optimal power for the FSA input can be derived as

$\int_{\Phi_{n} \lambda}^{\infty} p^{\star}\left(g_{n}, \mathcal{F}\right) h\left(g_{n}\right) d g_{n}=\frac{2}{d^{2} \sigma^{2}} \int_{\Phi_{n} \lambda}^{\infty} W\left(\alpha_{n} g_{n}{ }^{2}\right) e^{\frac{-g_{n}{ }^{2}}{2 \sigma^{2}}} d g_{n}$,

where $\alpha_{n}=\frac{U^{2} d^{2}}{2 \lambda^{2} \Phi_{n}^{2}}$. Using Taylor series, the right hand side of Eq. (26) becomes

$$
\begin{aligned}
& \approx \frac{2}{d^{2} \sigma^{2}}\left[A \int_{\Phi_{n} \lambda}^{\infty} e^{\frac{-g_{n} 2}{2 \sigma^{2}}} d g_{n}+B \int_{\Phi_{n} \lambda}^{\infty} g_{n} e^{\frac{-g_{n}{ }^{2}}{2 \sigma^{2}}} d g_{n}\right. \\
& \left.+C \int_{\Phi_{n} \lambda}^{\infty} g_{n}{ }^{2} e^{\frac{-g_{n}{ }^{2}}{2 \sigma^{2}}} d g_{n}+D \int_{\Phi_{n} \lambda}^{\infty} g_{n}{ }^{3} e^{\frac{-g_{n}{ }^{3}}{2 \sigma^{2}}} d g_{n}\right] .
\end{aligned}
$$

According to [15]

$$
\begin{aligned}
& \int_{u}^{\infty} x^{m} e^{-\beta x^{r}} d x=\frac{\Gamma\left(v, \beta u^{r}\right)}{r \beta^{v}}, \\
& \quad v=\frac{m+1}{r} \quad[\beta>0, \quad v>0, \quad r>0 \quad u>0] .
\end{aligned}
$$

A closed form of Eq. (27) can be derived as

$$
\begin{aligned}
& \approx \frac{\sqrt{2}}{d^{2} \sigma}\left[A \Gamma\left(\frac{1}{2}, \frac{\Phi_{n}^{2} \lambda^{2}}{2 \sigma^{2}}\right)+\sqrt{2} B \sigma \Gamma\left(1, \frac{\Phi_{n}^{2} \lambda^{2}}{2 \sigma^{2}}\right)\right. \\
& \left.+2 C \sigma^{2} \Gamma\left(\frac{3}{2}, \frac{\Phi_{n}^{2} \lambda^{2}}{2 \sigma^{2}}\right)+2 \sqrt{2} D \sigma^{3} \Gamma\left(2, \frac{\Phi_{n}^{2} \lambda^{2}}{2 \sigma^{2}}\right)\right] .
\end{aligned}
$$

By substituting Eq. (29) into Eq. (20), we obtain Eq. (18). To calculate A, B, C and D in Eq. (29), we need to derive $f(a), f^{\prime}(a), f^{\prime \prime}(a), f^{\prime \prime \prime}(a)$ by defining the function and taking its derivatives as follows

$$
\begin{gathered}
f\left(g_{\ell}\right)=W\left(\alpha_{\ell} g_{\ell}^{2}\right), \\
f^{\prime}\left(g_{\ell}\right)=\frac{2 W\left(\alpha_{\ell} g_{\ell}\right)}{g_{\ell}\left[W\left(\alpha_{\ell} g_{\ell}{ }^{2}\right)+1\right]}, \\
f^{\prime \prime}\left(g_{\ell}\right)=\frac{-2 W\left(\alpha_{\ell} g_{\ell}^{2}\right)\left[W\left(\alpha_{\ell} g_{\ell}^{2}\right)^{2}+W\left(\alpha_{\ell} g_{\ell}{ }^{2}\right)-1\right]}{g_{\ell}{ }^{2}\left[W\left(\alpha_{\ell} g_{\ell}{ }^{2}\right)+1\right]^{3}}, \\
f^{\prime \prime \prime}\left(g_{\ell}\right)=\frac{4 W\left(\alpha_{\ell} g_{\ell}^{2}\right)^{2}}{g_{\ell}^{3}\left[W\left(\alpha_{\ell} g_{\ell}^{2}\right)+1\right]^{5}} \\
{\left[W\left(\alpha_{\ell} g_{\ell}^{2}\right)^{3}+4 W\left(\alpha_{\ell} g_{\ell}^{2}\right)^{2}+3 W\left(\alpha_{\ell} g_{\ell}^{2}\right)-6\right] .}
\end{gathered}
$$

By substituting the values of $\alpha_{n}$ in Eqs. (30), (31), (32) and (33), A, B, C and D can be calculated. By substituting Eq. (25) into Eq. (20), the optimal power for Gaussian inputs can be derived as

$$
\begin{aligned}
\int_{\Phi_{n} \lambda}^{\infty} p^{\star}\left(g_{n}, G\right) h\left(g_{n}\right) d g_{n}= & \frac{1}{\sigma^{2} \lambda \Phi_{n}} \int_{\Phi_{n} \lambda}^{\infty} g_{n} e^{\frac{-g_{n}^{2}}{2 \sigma^{2}}} d g_{n} \\
& -\frac{1}{\sigma^{2}} \int_{\Phi_{n} \lambda}^{\infty} e^{\frac{-g_{n} 2}{2 \sigma^{2}}} d g_{n} .
\end{aligned}
$$

By applying Eq. (28), the RHS of Eq. (34) becomes

$$
=\frac{\Gamma\left(1, \frac{\Phi_{n}^{2} \lambda^{2}}{2 \sigma^{2}}\right)}{\lambda \Phi_{n}}-\frac{\Gamma\left(\frac{1}{2}, \frac{\Phi_{n}^{2} \lambda^{2}}{2 \sigma^{2}}\right)}{\sqrt{2} \sigma} .
$$

By substituting Eq. (35) into Eq. (20), we obtain Eq. (17).

\section{A. Theoretical Calculation of $\lambda$ for FSA and Gaussian Input} Distributions

In Eq. (18) and (17), $k_{n}, d$ and $\sigma$ are constant values, however, $\lambda$ is dependent on the channel gain. Therefore, we calculate $\lambda$ numerically via the following equation

$$
\sum_{n=1}^{N} \int_{\Phi_{n} \lambda}^{\infty} p^{\star}\left(g_{n}, S\right) \Phi_{n} h\left(g_{n}\right) d g_{n}=\frac{\tau_{t h}}{\Omega} .
$$

By substituting Eq. (24) into Eq. (36) and after the same manipulations as in Eqs. (26), (27) and (28), we can obtain the value of $\lambda$ for the FSA input using the following equation

$$
\begin{array}{r}
\frac{\sqrt{2} \Phi_{n}}{d^{2} \sigma} \sum_{n=1}^{N}\left[A \Gamma\left(\frac{1}{2}, \frac{\Phi_{n}^{2} \lambda^{2}}{2 \sigma^{2}}\right)+\sqrt{2} B \sigma \Gamma\left(1, \frac{\Phi_{n}^{2} \lambda^{2}}{2 \sigma^{2}}\right)\right. \\
\left.+2 C \sigma^{2} \Gamma\left(\frac{3}{2}, \frac{\Phi_{n}^{2} \lambda^{2}}{2 \sigma^{2}}\right)+2 \sqrt{2} D \sigma^{3} \Gamma\left(2, \frac{\Phi_{n}^{2} \lambda^{2}}{2 \sigma^{2}}\right)\right]=\frac{\tau_{t h}}{\Omega} .
\end{array}
$$

Similarly, by substituting Eq. (25) into Eq. (36) and after the same manipulations as in Eqs. (34) and (35), we can obtain the value of $\lambda$ for the Gaussian input using the following equation

$$
\sum_{n=1}^{N}\left[\frac{\Gamma\left(1, \frac{\Phi_{n}^{2} \lambda^{2}}{2 \sigma^{2}}\right)}{\lambda}-\frac{\Phi_{n} \Gamma\left(\frac{1}{2}, \frac{\Phi_{n}^{2} \lambda^{2}}{2 \sigma^{2}}\right)}{\sqrt{2} \sigma}\right]=\frac{\tau_{t h}}{\Omega} .
$$

By substituting the values of $k_{n}, N, \tau_{t h}, \Omega$ and $\sigma$ in Eq. (37) and Eq. (38) $\lambda$ can be calculated numerically.

The theoretical analysis gives deeper insights on the parameters affecting power saving. For example, it can be seen from Eq. (18) that the optimal power for FSA input distribution is inversely proportional to $d^{2}$. As $d(B P S K)>d(16-Q A M)$, therefore, average optimal power for BPSK is lower, leading to more power saving compared to the optimal power for 16QAM.

\section{Evaluation of OFDM BASED CR System}

\section{A. Simulation Analysis}

In Fig. 2, we compare $P_{G}^{\star}$ and $P_{\mathcal{F}}^{\star}$ versus distance. We observe from this figure that $P_{G}^{\star}$ is always greater than $P_{\mathcal{F}}^{\star}$ over the considered distance range. It has been noted that the gap is smaller at shorter distances compared to longer ones. The explanation is provided in [9]. Moreover, it has been observed that at a given distance, $P_{\mathcal{F}}^{\star}$ increases with the modulation order, (i.e., from BPSK to 16-QAM). The optimal power allocation is dependent and specific for every modulation scheme. It would result in power inefficiency if one tries to transmit BPSK signal with the power which is optimized for 16-QAM.

We have presented the results in [9] that the proposed optimal power allocation scheme achieves higher data rate compared to the Gaussian optimized power. We have shown that the percentage rate gain of the BPSK, QPSK and 16-QAM is $16.8-12.4 \%, 13-11.8 \%$ and $3-5.8 \%$, respectively, for the interference threshold values ranging between 1-3 $\mathrm{mW}$. However in this paper, we compare the achievable data rate for the FSA transmission under optimal power allocation with FSA inputs and uniform power loading scheme (i.e., $\left.\tau_{t h} /\left(\Omega \sum_{n=1}^{N} \Phi_{n}\right)\right)$ as shown in Fig. 4. It can be clearly 


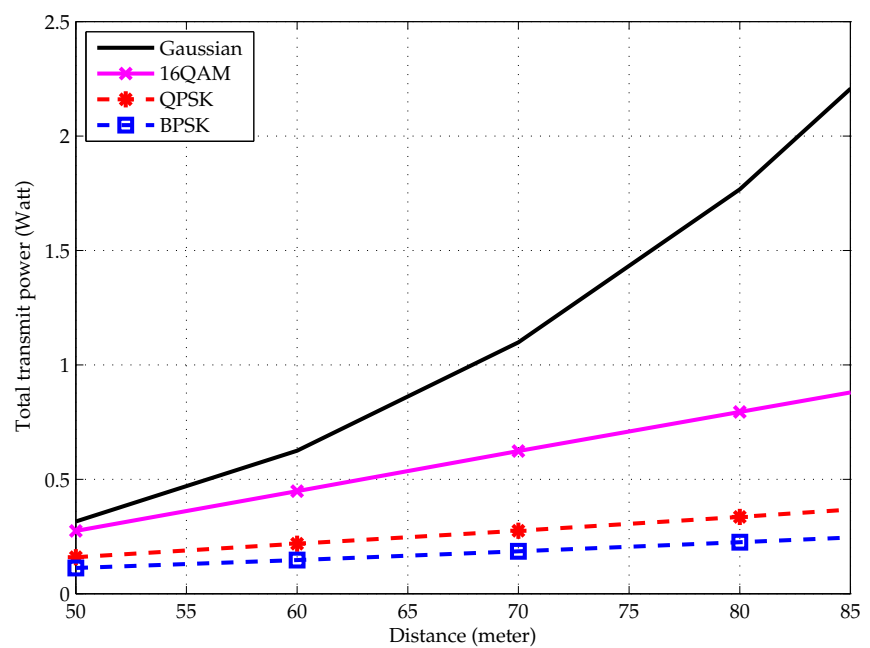

Fig. 2. Total transmit power under Gaussian and FSA inputs vs distance.

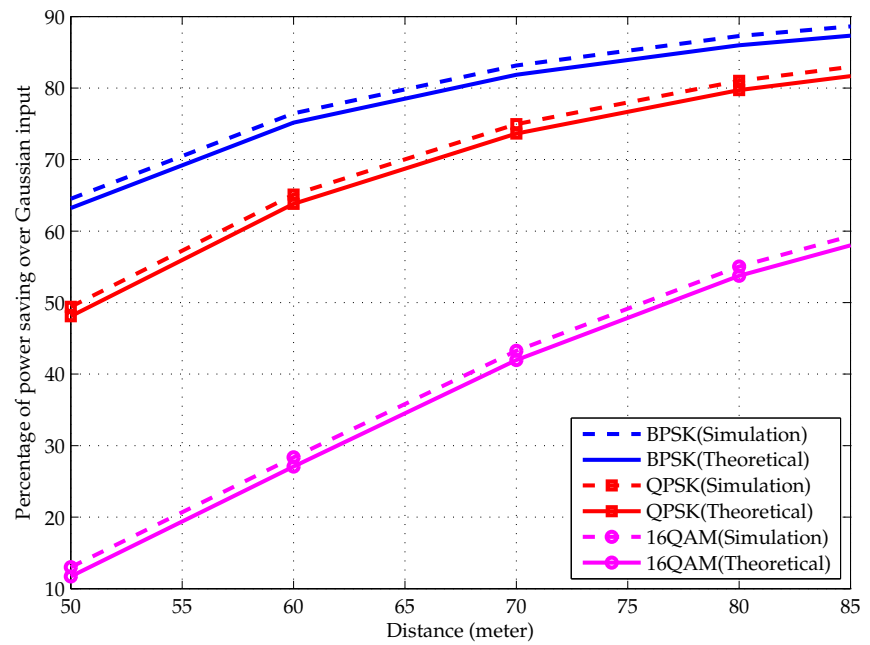

Fig. 3. Percentage of power saving vs distance.

seen that the proposed scheme outperforms the uniform power loading scheme over considered distance range.

Fig. 5 depicts the effect of normalized carrier frequency offset (i.e., $\epsilon$ ) on percentage of power saving over the Gaussian input by keeping the fixed distance $(60 \mathrm{~m})$. It has been observed that the percentage of power saving increases by increasing the values of $\epsilon$. This is due to the fact that the proposed optimal power and optimal power assuming the Gaussian input decreases by increasing the values of $\epsilon$, but the Gaussian optimized power decreases faster than the proposed optimal power. In Fig. 6, we compare percentage of power saving with the proposed and the conventional interference models. It has been shown that the percentage of power saving with the proposed interference model increases with the increased values of $\epsilon$, whereas, the percentage of power saving with the conventional interference model presented in [9] has a constant value because it does not depend upon values of $\epsilon$.

\section{B. Analytical Results vs. Simulation Results}

As discussed in Sec. III, our simulation study has shown that the proposed optimal power allocation scheme achieves

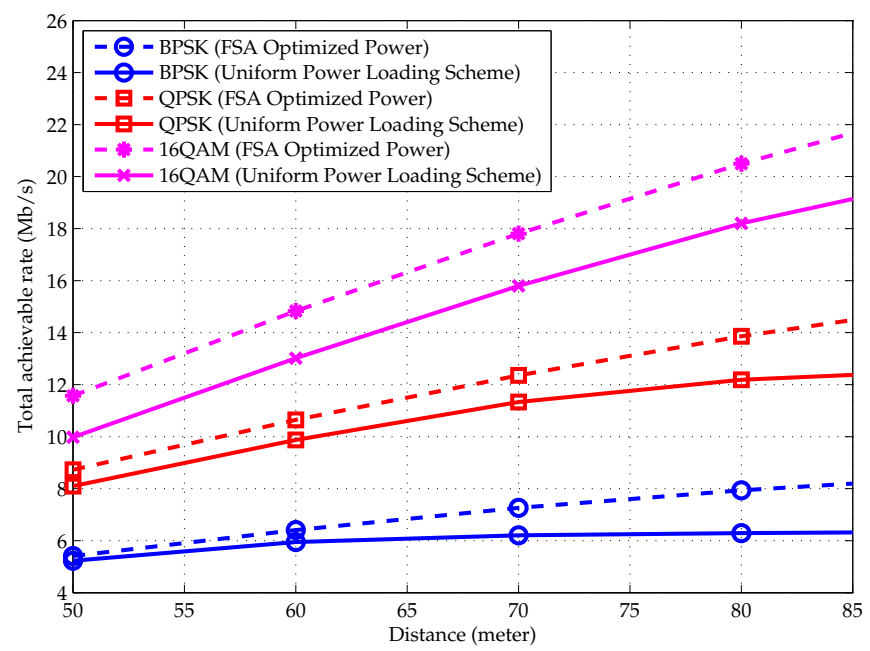

Fig. 4. Comparison of achievable data rate under FSA optimized power and uniform power loading scheme.

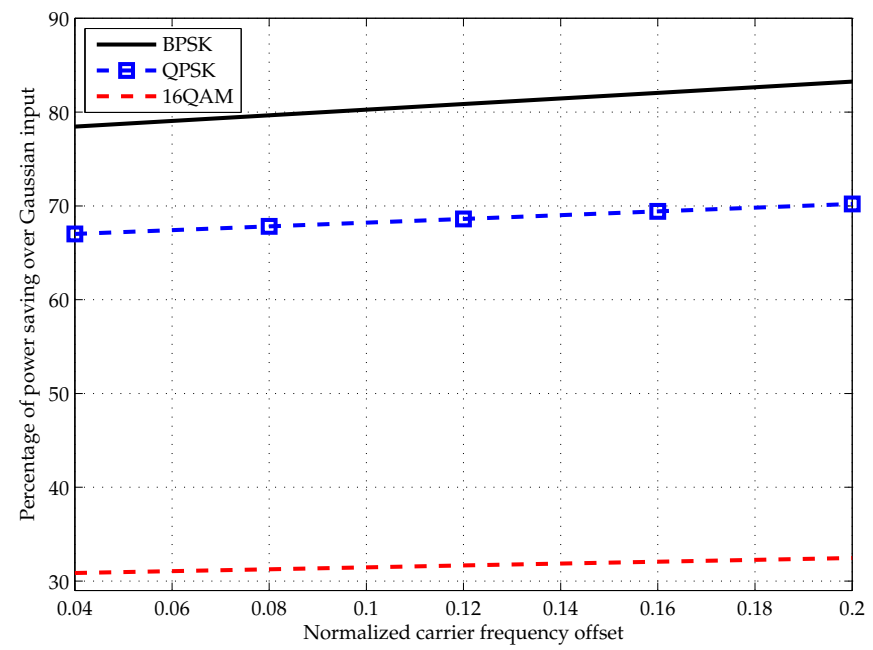

Fig. 5. Effect of $\epsilon$ on percentage of power saving at $60 \mathrm{~m}$ distance.

significant power saving compared to the optimal power under the Gaussian input. Fig. 3 shows the comparison of analytical (solid line) and simulated (dashed line) power saving. The channel static parameter, i.e., $\sigma$ in Eqs. (18) and (17) has been calculated from the empirical Rayleigh distribution and implemented in the simulation. One can see that theoretical results coincide well with the simulated ones, and the discrepancy is marginal. The minor difference follows from the fact that we used approximated values of MMSE in Eq. (21) and Taylor approximation in Eq. (26) to calculate the optimal power under the FSA input. It can be concluded that for given channel statistics, the theoretical analysis can be used to derive an average optimal power allocation and estimate power saving without running time consuming Monte Carlo simulations.

To evaluate the accuracy of using Taylor expansion, Fig. 7 depicts the optimal power of BPSK and the optimal power achieved by different degrees of Taylor polynomials. It is clear from the figure that the $5^{\text {th }}$ degree of Taylor polynomials approximately match the exact value and thus can be used to calculate the theoretical optimal power under arbitrary input 


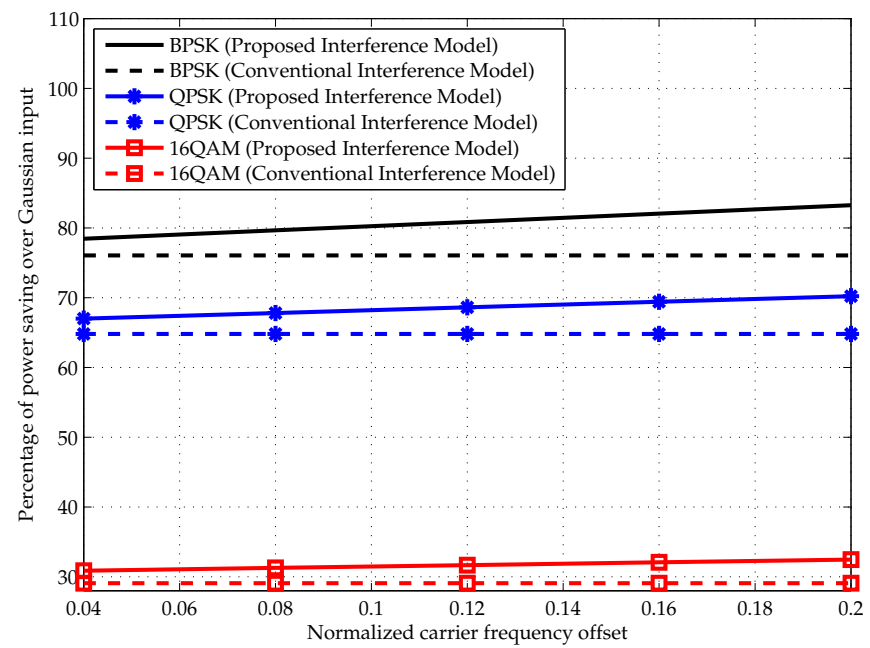

Fig. 6. Effect of $\epsilon$ on proposed and conventional interference models at $60 \mathrm{~m}$ distance.

distributions as well as the achieved power saving using the proposed power allocation scheme. The same accuracy of Taylor expansion has been noted for other modulation schemes in other figures.

\section{CONCLUSION}

In this paper, we first established the practical interference model that assumes OFDM modulation scheme for both the PU and the SU with imperfect synchronization. Accordingly, the power allocation problem in OFDM based CR systems is derived under the condition of FSA input applicable to practical systems. Motivated by the promising power saving result through Monte Carlo simulations, a theoretical evaluation of the power saving is presented in order to gain deeper insights into power saving capability of the proposed scheme. Furthermore, the theoretical results reveal that (i) our optimal power with the FSA input significantly outperforms the conventional power allocation schemes (i.e., the Gaussian optimized power and uniform power loading scheme) in terms of transmit power saving and achievable data rate; (ii) with fixed distance metric, the optimal transmit power with the FSA input increases as the modulation order increases, and (iii) by increasing the value of the normalized frequency offset $(\epsilon)$, the percentage of power saving increases with the proposed interference model, whereas, the percentage of power saving with the conventional interference model has a constant value. Based on the aforementioned findings we concluded that, by using the proposed power allocation scheme, spectrum and energy efficiency can both be improved. Secondly, in order to achieve a desired energy efficiency, the power should be optimized according to the employed modulation scheme.

\section{ACKNOWLEDGMENT}

This work has been supported by the India UK Advance Technology Centre of Excellence in Next Generation Networks, Systems and Services (www.iu-atc.com) and Engineering and Physical Sciences Research Council (EPSRC).

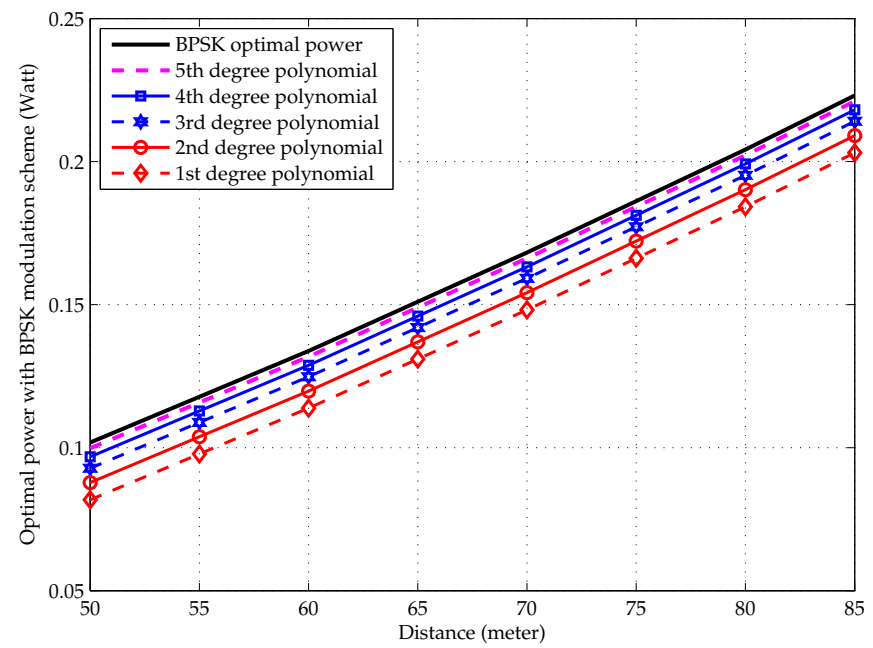

Fig. 7. Performance using Taylor series approximation.

\section{REFERENCES}

[1] E. Tragos, S. Zeadally, A. Fragkiadakis, and V. Siris, "Spectrum assignment in cognitive radio networks: A comprehensive survey," IEEE Commun. Surveys Tuts., vol. 15, no. 3, pp. 1108-1135, Third Quarter 2013.

[2] J. Wang, M. Ghosh, and K. Challapali, "Emerging cognitive radio applications: A survey," IEEE Commun. Mag., vol. 49, no. 3, pp. 74-81, Mar. 2011.

[3] V. Tumuluru, W. Ping, D. Niyato, and S. Wei, "Performance analysis of cognitive radio spectrum access with prioritized traffic," IEEE Trans. Veh. Technol., vol. 61, no. 4, pp. 1895-1906, May 2012.

[4] G. Bansal, J. Hossain, and V. Bhargava, "Optimal and suboptimal power allocation schemes for OFDM-based cognitive radio systems," IEEE Trans. Wireless Commun., vol. 7, no. 11, pp. 4710-4718, Nov. 2008.

[5] Z. Hasan, G. Bansal, E. Hossain, and V. Bhargava, "Energy-efficient power allocation in OFDM-based cognitive radio systems: A risk-return model," IEEE Trans. Wireless Commun., vol. 8, no. 12, pp. 6078-6088, Dec. 2009.

[6] C. Chiuan-Hsu and W. Chin-Liang, "Power allocation for OFDM-based cognitive radio systems under primary user activity," in IEEE Vehicular Technology Conference, May 2010, pp. 1-5.

[7] B. Devillers, J. Louveaux, and L. Vandendorpe, "Bit and power allocation for goodput optimization in coded parallel subchannels with ARQ," IEEE Trans. Signal Process., vol. 56, no. 8, pp. 3652-3661, Aug. 2008.

[8] A. Lozano, A. Tulino, and S. Verdu, "Optimum power allocation for parallel gaussian channels with arbitrary input distributions," IEEE Trans. Inf. Theory, vol. 52, no. 7, pp. 3033-3051, Jul. 2006.

[9] A. Sohail, M. Al-Imari, P. Xiao, and B. Evans, "Optimal power allocation for OFDM based cognitive radio systems with arbitrary input distributions," in IEEE Vehicular Technology Conference, Sep. 2013, pp. 1909-1913

[10] D. Guo, S. Shamai, and S. Verdu, "Mutual information and minimum mean-square error in Gaussian channels," IEEE Trans. Inf. Theory, vol. 51, no. 4, pp. 1261-1282, Apr. 2005.

[11] J. Zyren, "Overview of 3GPP long term evolution physical layer," Freescale Semiconductor, white paper, Jul. 2007.

[12] A. Goldsmith, Wireless Communications. Cambridge University Press, 2005.

[13] G. Arfken, "Incomplete Gamma function and related functions," Mathematical Methods for Physicists, pp. 565-572, 1985.

[14] R. M. Corless, G. H. Gonnet, D. E. G. Hare, D. J. Jeffrey, and D. E. Knuth, "On the Lambert W function," Advances in Computational Mathematics, vol. 5, no. 1, pp. 329-359, 1996.

[15] I. S. Gradshteyn and I. M. Ryzhik, Table of Integrals, Series and Products-7th Edition. Academic Press, 2007. 